\title{
Divide-and-Evolve: a Sequential Hybridization Strategy using Evolutionary Algorithms
}

\author{
Marc Schoenauer $^{1}$, Pierre Savéant ${ }^{2}$, and Vincent Vidal ${ }^{3}$ \\ 1 Projet TAO, INRIA Futurs, LRI, Bt. 490, Université Paris Sud, 91405 Orsay, \\ France marc.schoenauer@inria.fr \\ 2 Thales Research \& Technology France, RD 128, F-91767 Palaiseau, France \\ pierre.saveant@thalesgroup.com \\ 3 CRIL \& Université d'Artois, rue de l'université - SP16, 62307 Lens, France \\ vidal@cril.univ-artois.fr
}

\begin{abstract}
Summary. Memetic Algorithms are hybridizations of Evolutionary Algorithms (AEs) with problem-specific heuristics or other meta-heuristics, that are generally used within the EA to locally improve the evolutionary solutions. However, this approach fails when the local method stops working on the complete problem. Divideand-Evolve is an original approach that evolutionarily builds a sequential slicing of the problem at hand into several, hopefully easier, sub-problems: the embedded (meta-)heuristic is only asked to solve the 'small' problems, and Divide-and-Evolve is thus able to globally solve problems that are intractable when directly fed into the heuristic. The Divide-and-Evolve approach is described here in the context of Temporal Planning Problems (TPPs), and the results on the standard Zeno transportation benchmarks demonstrate its ability to indeed break the complexity barrier. But an even more prominent advantage of the Divide-and-Evolve approach is that it immediately opens up an avenue for multi-objective optimization, even when using single-objective embedded algorithm.
\end{abstract}

Key words: Hybrid Algorithms, Temporal Planning, Multi-Objective Optimization

\section{Introduction}

Evolutionary Algorithms (EAs) are bio-inspired meta-heuristics crudely borrowing to the Darwinian theory of natural evolution of biological populations (see [7] for the most recent comprehensive introduction, or [6] for a brief introduction of the basic concepts). In order to solve the optimization problem at hand, EAs evolve a population of individuals (tuples of candidate solutions) relying on two main driving forces to reach the optimal solution: natural selection and blind variations. Natural selection biases the choices of the algorithm toward good performing individuals (w.r.t. the optimization problem at 
hand), at reproduction time and at survival time (survival of the fittest). Blind variation operators are stochastic operators defined on the search space that create new individuals from parents in the current population, independently of their performance (hence the term 'blind'). They are usually categorized into crossovers, producing offspring from 2 parents, and mutations that create one offspring from a single parent. Whereas the natural selection part of an EA is (almost) problem independent, the choice of the search space (the representation) and the corresponding variation operators has to be done anew for each application domain, and requires problem-specific expertise.

This has been clearly demonstated in the domain of Combinatorial Optimization, where it is now well-known (see Grefensetette's seminal paper [12]) that generic EAs alone are rarely efficient. However, the flexibility of EAs allows the user to easily add domain knowledge at very different levels of the algorithm, from representation [23] to ad hoc variation operators [31] to explicit use of other optimization techniques within the EA: The most successful of such hybridizations indeed use other heuristics or meta-heuristics to locally improve all individuals that are created by the EA, from the initial population to all offspring that are generated by the variation operators. Such algorithms have been termed "Memetic Algorithms" or "Genetic Local Search" [22]. Those methods are now the heart of a very active research field, as witnessed by the yearly WOMA series (Workshops on Memetic Algorithms), Journal Special Issues [13] and edited books [14].

However, most memetic approaches are based on finding local improvements of candidate solutions proposed by the evolutionary search mechanism using dedicated local search methods that have to tackle the complete problem. Unfortunately, in many combinatorial domains, this simply proves to be impossible when reaching some level of complexity. This paper proposes an original hybridization of EAs with a domain-specific solver that addresses this limitation in domains where the task at hand can be sequentially decomposed into a series of (hopefully) simpler tasks. Temporal Planning is such a domain, that will be used here to instanciate the Divide-and-Evolve paradigm.

Artificial Intelligence Planning is a form of general problem solving task which focuses on problems that map into state models that can be defined by a state space $S$, an initial state $s_{0} \subseteq S$, a set of goal states $S_{G} \subseteq S$, a set of actions $A(s)$ applicable in each state $S$, and a transition function $f(a, s)=s^{\prime}$ with $a \in A(s)$, and $s, s^{\prime} \in S$. A solution to this class of models is a sequence of applicable actions mapping the initial state $s_{0}$ to a goal state that belongs to $S_{G}$.

An important class of problems is covered by Temporal Planning which extends classical planning by adding a duration to actions and by allowing concurrent actions in time [11]. In addition, other metrics are usually needed for real-life problems to qualify a good plan, for instance a cost or a risk criterion. A usual approach is to aggregate the multiple criteria, but this relies on highly problem-dependent features and is not always meaningful. A 
better solution is to compute the set of optimal non-dominated solutions the so-called Pareto front.

Because of the high combinatorial complexity and the multi-objective features of Temporal Planning Problems (TPPs), Evolutionary Algorithms seem to be good general-purpose candidate methods.

However, there has been very few attempts to apply Evolutionary Algorithms to Planning Problems and, as far as we know, not any to Temporal Planning. Some approaches use a specific representation (e.g. dedicated to the battlefield courses of action [24]). Most of the domain-independent approaches see a plan as a program and rely on Genetic Programming and on the traditional blocks-world domain for experimentation (starting with the Genetic Planner [27]). A more comprehensive state of the art on Genetic Planning can be found in [2] where the authors experimented a variable length chromosome representation. It is important to notice that all those works search the space of (partial) plans.

In this context, the Divide-and-Evolve approach, borrowing to the Divideand-Conquer paradigm, tries to slice the problem at hand into a sequence of problems that are hopefully easier to solve by the available OR or local methods. The solution to the original problem is then obtained by a concatenation of the solutions to the different sub-problems.

Note that the idea to divide the plan trajectory into small chunks has been studied with success in [15]. The authors have shown the existence of landmarks, i.e. sets of facts that must be true at some point during execution of any solution plan, and the impact of ordering them on search efficiency. They also prove that deciding if a fact is a landmark and finding ordering relations is PSPACE-complete. In this way, Divide-and-Evolve can be seen as an attempt to generate ordered sets of landmarks using a stochastic approach. However, the proposed approach is not limited to finding sets of facts that must absolutely be true within every solution to the initial problem. In particular, it also applies to problems that have no landmark per se, for simple symmetry reasons: there can be several equivalent candidate landmarks, and only one of them can and must be true at some point.

The chapter is organized as follows: Next section presents an abstract formulation of the Divide-and-Evolve scheme, and starting from its historical (and pedagogical) root, the TGV paradigm. Generic representation and variation operators are also introduced. Section 3 introduces an actual instantiation of the Divide-and-Evolve scheme to TPPs. The formal framework of TPPs is first introduced, then the TPP-specific issues for the Divide-and-Evolve implementation are presented and discussed. Section 4 is devoted to experiments on the TPP transportation Zeno benchmark for both single and multi-objective cases. The local problems are solved using the exact temporal planner CPT [28], a freely-available optimal temporal planner, for its temporal dimension. The last section opens a discussion highlighting the limitations of the present 
work and sketching further directions of research.

Note that an initial presentation of Divide-and-Evolve was published at EvoCOP'06 conference [25], in which only very preliminary results were presented in the single-objective case. Those results are here validated more thoroughly on the 3 instances Zeno10, Zeno12 and Zeno14: the new experiments demonstrate that the Divide-and-Evolve approach can repeatedly find the optimal solution on all 3 instances. Moreover, the number of backtracks that are needed by the optimal planner CPT to solve each sub-problem is precisely analyzed, and it is demonstrated that it is possible to find the optimal solution even when limiting the number of backtracks that CPT is allowed to perform for each sub-problem, thus hopefully speeding up the complete optimization. On the other hand, however, the multi-objective results that are presented here are the same than those of [25], and are recalled here for the sake of completeness, as they represent, as far as we know, the very first results of Pareto multi-objective optimization in Temporal Planning, and open up many avenues for further research.

\section{The Divide-and-Evolve Paradigm}

This section presents the Divide-and-Evolve scheme, an abstraction of the TGV paradigm that can be used to solve a planning problem when direct methods face a combinatorial explosion due to the size of the problem. The TGV approach might be a way to break the problem into several subproblems, hopefully easier to solve than the initial global problem.

\subsection{The TGV metaphor}

The Divide-and-Evolve strategy springs from a metaphor on the route planning problem for the French high-speed train (TGV). The original problem consists in computing the shortest route between two points of a geographical landscape with strong bounds on the curvature and slope of the trajectory. An evolutionary algorithm was designed [5] based on the fact that the only local search algorithm at hand was a greedy deterministic algorithm that could solve only very simple (i.e. short distance) problems. The evolutionary algorithm looks for a split of the global route into small consecutive segments such that a local search algorithm can easily find a route joining their extremities. Individuals represent sets of intermediate train stations between the station of departure and the terminus. The convergence toward a good solution was obtained with the definition of appropriate variation and selection operators [5]. Here, the state space is the surface on which the trajectory of the train is defined. 


\section{Generalization}

Abstracted to Planning Problems, the route is replaced by a sequence of actions and the "stations" become intermediate states of the system. The problem is thus divided into sub-problems and "to be close" becomes "to be easy to solve" by some local algorithm $\mathcal{L}$. The evolutionary algorithm plays the role of an oracle pointing at some imperative states worth to go trough.

\subsection{Representation}

The problem at hand is an abstract AI Planning problem as described in the introduction. The representation used by the evolutionary algorithm is a variable length list of states: an individual is thus defined as $\left(s_{i}\right)_{i \in[1, n]}$, where the length $n$ and all the states $s_{i}$ are unknown and subject to evolution. States $s_{0}$ and $s_{n+1} \equiv s_{G}$ will represent the initial state and the goal of the problem at hand, but will not be encoded in the genotypes. By reference to the original TGV paradigm, each of the states $s_{i}$ of an individual will be called a station.

\section{Requirements}

The original TGV problem is purely topological with no temporal dimension and reduces to a planning problem with a unique action: moving between two points. The generalization to a given planning domain requires to be able to:

1. define a distance between two different states of the system, so that $d(S, T)$ is somehow related to the difficulty for the local algorithm $\mathcal{L}$ to find a plan mapping the initial state $S$ to the final state $T$;

2. generate a chronological sequence of virtual "stations", i.e. intermediate states of the system, that are close to one another, $s_{i}$ being close to $s_{i+1}$;

3. solve the resulting "easy" problems using the local algorithm $\mathcal{L}$;

4. "glue" the sub-plans into an overall plan of the problem at hand.

\subsection{Variation operators}

This section describes several variation operators that can be defined for the general Divide-and-Evolve approach, independently of the actual domain of application (e.g. TPPs, or the original TGV problem).

\section{Crossover}

Crossover operators amounts to exchanging stations between two individuals. Because of the sequential nature of the fitness, it seems a good idea to try to preserve sequences of stations, resulting in straightforward adaptations to variable-length representation of the classical 1- or 2-point crossover operators. 
Suppose you are recombining two individuals $\left(s_{i}\right)_{1 \leq n}$ and $\left(T_{i}\right)_{1 \leq m}$. The 1 -point crossover amounts to choosing one station in each individual, say $s_{a}$ and $T_{b}$, and exchanging the second part of the lists of stations, obtaining the two offspring $\left(s_{1}, \ldots, s_{a}, T_{m+1}, \ldots T_{b}\right)$ and $\left(T_{1}, \ldots, T_{b}, s_{n+1}, \ldots, s_{n}\right)$ (2-point crossover is easily implemented in a similar way). Note that in both cases, the length of each offspring is likely to differ from those of the parents.

The choice of the crossover points $s_{a}$ and $T_{b}$ can be either uniform (as done in all the work presented here), or distance-based, if some distance is available: pick the first station $s_{a}$ randomly, and choose $T_{b}$ by e.g. a tournament based on the distance with $s_{a}$ (this is on-going work).

\section{Mutation}

Several mutation operators can be defined. Suppose individual $\left(s_{i}\right)_{1 \leq n}$ is being mutated:

- At the individual level, the $A d d$ mutation simply inserts a new station $s_{\text {new }}$ after a given station $\left(s_{a}\right)$, resulting in an $n+1$-long list, $\left(s_{1}, \ldots, s_{a}\right.$, $\left.s_{\text {new }}, s_{a+1}, \ldots, s_{n}\right)$. Its counterpart, the Del mutation, removes a station $s_{a}$ from the list.

Several improvements on the pure uniform choice of $s_{a}$ can be added and are part of on-going work, too: in case the local algorithm fails to successfully join all pairs of successive stations, the last station that was successfully reached by the local algorithm can be preferred for station $s_{a}$ (in both the $A d d$ and Del mutations). If all partial problems are solved, the most difficult one (e.g. in terms of number of backtracks) can be chosen.

- At the station level, the definition of each station can be modified but this is problem-dependent. However, assuming there exists a stationmutation operator $\mu_{S}$, it is easy to define the individual-mutation $M_{\mu_{S}}$ that will simply call $\mu_{S}$ on each station $s_{i}$ with a user-defined probability $p_{\mu_{S}}$. Examples of operators $\mu_{S}$ will be given in section 3 , while simple Gaussian mutation of the $(x, y)$ coordinates of a station were used for the original TGV problem [5].

\section{Application to Temporal Planning}

\subsection{Temporal planning problems}

Domain-Independent planners rely on the Planning Domain Definition Language (PDDL) [20], inherited from the STRIPS model [8], to represent a planning problem. In particular, this language is used for a competition (see http://ipc.icaps-conference.org/) which is held every two years since $1998[21,1,19,16]$. The language has been extended for representing Temporal Planning Problems in PDDL2.1 [10]. For the sake of simplicity, and 
because the underlying temporal planner that we use, CPT $[28,29]$, is not strictly conformant to PDDL2.1, the temporal model is often simplified as explained below [28].

A Temporal PDDL Operator is a tuple $o=\langle\operatorname{pre}(o), \operatorname{add}(o), \operatorname{del}(o), \operatorname{dur}(o)\rangle$ where $\operatorname{pre}(o), \operatorname{add}(o)$ and $\operatorname{del}(o)$ are sets of ground atoms that respectively denote the preconditions, add effects and del effects of $o$, and $\operatorname{dur}(o)$ is a rational number that denotes the duration of $o$. The operators in a PDDL input can be described with variables, used in predicates such as (at ?plane ?city). The variables ?plane and ?city are then replaced by CPT with the objects of a particular problem in an initial grounding process.

A Temporal Planning Problem is a tuple $P=\langle A, I, O, G\rangle$, where $A$ is a set of atoms representing all the possible facts in a world situation, $I$ and $G$ are two sets of atoms that respectively denote the initial state and the problem goals, and $O$ is a set of ground PDDL operators.

As is common in Partial Order Causal Link (POCL) Planning [30], two dummy actions are also considered, Start and End with zero durations, the first with an empty precondition and effect $I$; the latter with precondition $G$ and empty effects. Two actions $a$ and $a^{\prime}$ interfere when one deletes a precondition or positive effect of the other. The simple model of time in [26] defines a valid plan as a plan where interfering actions do not overlap in time. In other words, it is assumed that the preconditions need to hold until the end of the action, and that the effects also hold at the end and cannot be deleted during the execution by a concurrent action.

A schedule $P$ is a finite set of actions occurrences $\left\langle a_{i}, t_{i}\right\rangle, i=1, \ldots, n$, where $a_{i}$ is an action and $t_{i}$ is a non-negative integer indicating the starting time of $a_{i}$ (its ending time is $\left.t_{i}+\operatorname{dur}\left(a_{i}\right)\right)$. $P$ must include the Start and End actions, the former with time tag 0 . The same action (except for these two) can be executed more than once in $P$ if $a_{i}=a_{j}$ for $i \neq j$. Two action occurrences $a_{i}$ and $a_{j}$ overlap in $P$ if one starts before the other ends; namely if $\left[t_{i}, t_{i}+\operatorname{dur}\left(a_{i}\right)\right] \cap\left[t_{j}, t_{j}+\operatorname{dur}\left(a_{j}\right)\right]$ contains more than one time point.

A schedule $P$ is a valid plan iff interfering actions do not overlap in $P$ and for every action occurrence $\left\langle a_{i}, t_{i}\right\rangle$ in $P$ its preconditions $p \in \operatorname{pre}(a)$ are true at time $t_{i}$. This condition is inductively defined as follows: $p$ is true at time $t=0$ iff $p \in I$, and $p$ is true at time $t>0$ if either $p$ is true at time $t-1$ and no action $a$ in $P$ ending at $t$ deletes $p$, or some action $a^{\prime}$ in $P$ ending at $t$ adds $p$. The makespan of a plan $P$ is the time tag of the End action.

\subsection{CPT: an optimal temporal planner}

An optimal temporal planner computes valid plans with minimum makespan. Even though an optimal planner was not mandatory (as discussed in section 5), we have chosen $C P T$ [28], a freely-available optimal temporal planner, for its temporal dimension and for its constraint-based approach which provide a very useful data structure when it comes to gluing the partial solutions (see section 2.2). Indeed, since in Temporal Planning actions can overlap in time, 
the simple concatenation of sub-plans, though providing a feasible solution, obviously might produce a plan that is not optimal with respect to the total makespan, even if the sequence of actions is the optimal sequence. However, thanks to the causal links and order constraints maintained by CPT, an improved global plan can be obtained by shifting sub-plans as early as possible in a final state of the algorithm.

Another argument for choosing CPT was the fact that it is a sound and complete planner in the following sense: a valid plan with makespan equal to a given bound $B$ on the number of allowed backtracks is found if and only if one such plan exists. There are then many strategies for adjusting the bound $B$ so that an optimal makespan is produced; e.g., the bound may be increased until a plan is found, or can be decreased until no plan is found, etc.

Indeed, because one motivation for the Divide-and-Evolve approach is to tackle large instances that are too complex to be directly solved by the local algorithm (CPT in our case), it is important to be able to launch only limited searches by CPT: a bound on the number of allowed backtracks could be added to all CPT calls, and the fitness was penalized when this bound was reached (CPT stops without giving a result in that case). More details will be given in section 4.1 .

One final reason for originally choosing CPT, before the collaboration among the authors of this work started, was that a binary version was freely available on the third author's Web page. However, even though a tighter collaboration rapidly became effective, it proved nevertheless intractable to call CPT as a subroutine, for technical reasons (CPT-2 was written in CLAIRE). Hence data had to be passed through files, and CPT launched anew each time, resulting in a huge waste of CPU resources. This drawback will be solved by switching to CPT-3, the most recent version of CPT (on-going work).

\subsection{Rationale for using Divide-and-Evolve for Temporal Planning}

The reasons for the failure of standard OR methods addressing TPPs come from the exponential complexity of the number of possible actions when the number of objects involved in the problem increases. It is known for a long time that taking into account the interactions between sub-goals can decrease the complexity of finding a plan, in particular when these sub-goals are independent [18]. Moreover, computing an ideal ordering on sub-goals is as difficult as finding a plan (PSPACE-hard), as demonstrated in [17]. which proposes an algorithm for computing an approximation of such an ordering. The basic idea when using the Divide-and-Evolve approach is that each local sub-plan ("joining" stations $s_{i}$ and $s_{i+1}$ ) should be easier to find than the global plan (joining the station of departure $s_{0}$ and the terminus $s_{n+1}$ ). This will be now demonstrated on the Zeno transportation benchmark (see http://ipc.icaps-conference.org/).

Table 1 illustrates the decomposition of a relatively difficult problem in the Zeno domain (zeno14 from IPC-3 benchmarks), a transportation problem 
with 5 planes (plane1 to plane5) and 10 persons (person1 to person10) to travel among 10 cities (city0 to city9). A plane can fly at two different speeds. Flying fast requires more fuel. A plan has a fuel level and might be refueled when empty. A person is either at a city or in a plane and requires to be boarded and disembarked.

Analyzing the optimal solution found by CPT-3, it was possible (though not trivial) to manually divide the optimal "route" of this solution in the state space into four intermediate stations between the initial state and the goal. It can be seen that very few moves (plane or person) occur between two consecutive stations (the ones in bold in each column of Table 1). Each sub-plan is easily found by CPT, with a maximum of 1 backtrack and 1.87 seconds of search time. It should be noted that most of the time spent by CPT is for pre-processing: this operation is actually repeated each time CPT is called, but could be factorized at almost no cost ... except coding time.

Note that the final step of the process is the compression of the five subplans (see section 2.2): it is here performed in 0.10 seconds (plus 30.98 seconds for pre-processing) without any backtracking, and the overall makespan of the plan is 476 , much less than the sum of the individual makespans of each subplan (982).

To summarize, the recomposed plan, with a makespan of 476 , required a total running time of 193.30 seconds (including only 7.42s of pure search) and only one backtrack, whereas a plan with the same optimal makespan of 476 is found by CPT in 2,692.41 seconds and 566,681 backtracks. Section 5 will discuss this issue.

\subsection{Description of the state space}

\section{Non-temporal states}

A natural state space for TPPs, as described at the beginning of this section, would be the actual space of all possible time-stamped states of the system. Obviously, the size of such a space is far too big and we simplified it by restricting the stations to non-temporal states. However, even with this simplification, not all "non-temporal" states can be considered in the description of the "stations".

\section{Limiting the possible states}

First, the space of all possible states grows exponentially with the size of the problem. Second, not all states are consistent w.r.t. the planning domain. For instance, an object cannot be located at two places at the same time in a transportation problem - and inferring such state invariants is feasible but not trivial [9]. Note also that determining plan existence from a propositional STRIPS description has been proved to be PSPACE-complete [3]. 
Table 1. State Decomposition of the Zeno14 Instance. (The new location of moved objects appears in bold.)

\begin{tabular}{|c|c|c|c|c|c|c|}
\hline Objects & $\begin{array}{c}\text { Init } \\
(\text { station } 0)\end{array}$ & Station 1 & Station 2 & Station 3 & Station 4 & $\begin{array}{c}\text { Goal } \\
\text { (station 5) }\end{array}$ \\
\hline plane 1 & city 5 & city 5 & city 5 & city 6 & city 6 & city 6 \\
\hline plane 2 & city 2 & city 0 & city 0 & city 2 & city 3 & city 3 \\
\hline plane 3 & city 4 & city 7 & city 9 & city 7 & city 7 & city 9 \\
\hline plane 4 & city 8 & city 8 & city 8 & city 7 & city 5 & city 5 \\
\hline plane 5 & city 9 & city 6 & city 1 & city 1 & city 8 & city 8 \\
\hline person 1 & city 9 & city 9 & city 9 & city 9 & city 9 & city 9 \\
\hline person 2 & city 1 & city 1 & city 1 & city 1 & city 8 & city 8 \\
\hline person 3 & city 0 & city 0 & city 0 & city 2 & city 2 & city 2 \\
\hline person 4 & city 9 & city 9 & city 9 & city 7 & city 7 & city 7 \\
\hline person 5 & city 6 & city 6 & city 1 & city 1 & city 1 & city 1 \\
\hline person 6 & city 0 & city 0 & city 0 & city 6 & city 6 & city 6 \\
\hline person 7 & city 7 & city 7 & city 7 & city 7 & city 5 & city 5 \\
\hline person 8 & city 6 & city 6 & city 1 & city 1 & city 1 & city 1 \\
\hline person 9 & city 4 & city 7 & city 7 & city 7 & city 5 & city 5 \\
\hline person 10 & city 7 & city 7 & city 9 & city 9 & city 9 & city 9 \\
\hline \multicolumn{2}{|c|}{ Makespan } & 150 & 203 & 150 & 276 & 203 \\
\hline \multicolumn{2}{|c|}{ Backtracks } & 0 & 0 & 0 & 1 & 0 \\
\hline \multirow{2}{*}{\multicolumn{2}{|c|}{$\begin{array}{l}\text { Search time } \\
\text { Total time }\end{array}$}} & 1.34 & 1.27 & 1.32 & 1.87 & 1.52 \\
\hline & & 32.32 & 32.25 & 32.30 & 32.85 & 32.50 \\
\hline \multicolumn{2}{|c|}{ Total time } & \multicolumn{2}{|c|}{ Compression } & & \multicolumn{2}{|c|}{ Global Search } \\
\hline \multicolumn{2}{|c|}{ Makespan } & \multicolumn{2}{|c|}{476} & & \multicolumn{2}{|c|}{476} \\
\hline \multicolumn{2}{|c|}{ Backtracks } & \multicolumn{2}{|c|}{0} & & \multicolumn{2}{|c|}{566,681} \\
\hline \multicolumn{2}{|c|}{ Search time } & \multicolumn{2}{|c|}{0.10} & & \multicolumn{2}{|c|}{$2,660.08$} \\
\hline \multicolumn{2}{|c|}{ Total time } & \multicolumn{2}{|c|}{31.08 (total : 193.30) } & & \multicolumn{2}{|c|}{$2,692.41$} \\
\hline
\end{tabular}

A possible way to overcome this difficulty would be to rely on the local algorithm to (rapidly) check the consistency of a given situation, and to penalize unreachable stations. However, this would clearly be a waste of computational resources, possibly leading to a far too difficult problem to solve for the EA (it would have to "discover" again and again that one object cannot be at the same time at two different locations, without even a way to somehow generalize and save this across different situations).

On the other hand, introducing domain knowledge into EAs has been known for long as the royal road toward success in Evolutionary Computation [12]. Hence, it seems a more promising approach to add state invariants to the description of the state space in order to remove the inconsistent states as much as possible. The good thing is that it is not necessary to remove all inconsistent states since, in any case, the local algorithm is there to help the EA to spot them - inconsistent stations will be given poor fitness, and will not survive next selection steps. In particular, only state invariants involving a single predicate have been implemented in the present work. 


\subsection{Representation of stations}

It was hence decided to describe the stations using only the predicates that are present in the goal of the overall problem, and to maintain the state invariants based on the semantics of the problem.

A good example is given in Table 1: the goal of this benchmark instance is to move the persons and planes in cities listed in the last column. No other predicate than the corresponding (at objectN cityM) predicates is present in the goal. Through a user-supplied file, the algorithm is told that only the at predicates will be used to represent the stations, with the syntactic restrictions that within a given station, the first argument of an at predicate can appear only once (at is said to be exclusive with respect to its first argument). The state space that will be explored by the algorithm thus amounts to a vector of 15 fluents (instantiated predicates) denoting that an item is located in a city (a column of table 1). In addition, the actual implementation of a station includes the possibility to "remove" (in fact, comment out) a predicate of the list: the corresponding object will not move during this sub-plan.

\section{Distance}

The distance between two stations should reflect the difficulty for the local algorithm to find a plan joining them. At the moment, a purely syntactic domain-independent distance is used: the number of different predicates not yet reached. The difficulty can then be estimated by the number of backtracks needed by the local algorithm. It is reasonable to assume that indeed most local problems where only a few predicates need to be changed from the initial state to the goal will be easy for the local algorithm - though this is certainly not true in all cases.

\section{Random generation of stations}

Thanks to the state invariants described above, generating random stations now amounts to choose among consistent stations, and is thus rather simple for a single station. Nevertheless, the generation of initial individuals (sequences of stations $\left(s_{i}\right)_{i \in[1, n]}$ such that all local problems $\left(s_{i}, s_{i+1}\right)$ are simple for the local algorithm) remains an issue. A very representation-specific method has been used for TPPs that will be described in next section.

\subsection{Representation-specific operators}

The initialization of an individual (see section 3.5) and the station-mutation operator (see section 2.3) will be described for the chosen problem-specific representation for TPPs. 


\section{Initialization}

First, the number of stations is chosen uniformly in a user-supplied interval. The user also enters a maximal distance $d_{\max }$ between stations: two consecutive stations will not differ by more that $d_{\max }$ predicates. (the minimal number of stations is eventually adjusted in order to meet the requirement, according to the distance between the initial state and the goal state). A matrix is then built, similar to the top lines of table 1: each line corresponds to one of the goal predicates, each column is a station. Only the first and last columns (corresponding to initial state and goal) are filled with values. A number of "moves" is then randomly added in the matrix, at most $d_{\max }$ per column, and at least one per line. Additional moves are then added according to another user-supplied parameter, and without exceeding the $d_{\max }$ limit per column. The matrix is then filled with values, starting from both ends (init and goal), constrained column-wise by the state invariants, as described in section 3.4 and line-wise by the values in the init and goal states. If some station proves to be inconsistent at some point, it is rejected and a new one is generated. A final sweep on all predicates comments out some of the predicates with a given probability.

\section{Station mutation}

Thanks to the simplified representation of the states (a vector of fluents with a set of state invariants), it is straightforward to modify one station randomly: with a given probability, a new value for the non-exclusive arguments is chosen among the possible values respecting all constraints (including the distance constraints with previous and next stations). In addition, each predicate might be commented out from the station with a given probability, like in the initialization phase.

\section{First Experiments}

\subsection{Single objective optimization}

Our main playground to validate the Divide-and-Evolve approach is that of transportation problems, and started with the zeno domain as described in section 3.3. As can be seen in table 1, the description of the stations in zeno domain involves a single predicate, at, with two arguments. It is exclusive w.r.t. its first argument. Three instances have been tried, called zeno10, zeno12 and zeno14, from the simplest to the hardest.

The simple zeno10 (resp. zeno12) instance can be solved very easily by CPT-2 alone, in less than 2s (resp. 125s), finding the optimal plans with makespan 453 (resp. 549) using 154 (resp. 27560) backtracks. On the other hand, the zeno14 instance could not be solved at all by CPT-2. However, as described in Table 1, the new version CPT-3 could solve it, with a makespan of 476 and using 566,681 backtracks. 


\section{Algorithmic settings}

The EA that was used for the first implementation of the Divide-and-Evolve paradigm use standard algorithmic settings at the population level:

- Population size was set to 10 to limit the CPU cost

- both a $(10+10)-E S$ and a $(10,70)-E S$ evolution engines were used: the 10 parents give birth to either 10 or 70 children, and the best 10 among the 10 children plus the 10 parents $((10+10)-E S)$ or among the 70 children $((10,70)-E S)$ become the parents of next generation;

- 1-point crossover is applied to $25 \%$ of the individuals

- the other $75 \%$ undergo mutation: $25 \%$ of the mutations are the Add (resp. Del) generic mutations (section 2.3). The remaining $50 \%$ of the mutations call the problem-specific station mutation. Within a station mutation, a predicate is randomly changed in $75 \%$ of the cases and a predicate is removed (resp. restored) in each of the remaining $12.5 \%$ cases. (see section 3.6).

- Initialization is performed using initial size in $[2,10]$, maximum distance of 3 and probability to comment out a predicate is set to 0.1 .

Note that at the moment, no lengthy parameter tuning was performed for those proof-of-concept experiments, and the above values were decided based upon a very limited set of initial experiments.

\section{The fitness}

The target objective is here the total makespan of a plan - assuming that a global plan can be found, i.e. that all problems $\left(s_{i}, s_{i+1}\right)$ can be solved by the local algorithm. In case one of the local problems could not be solved, the individual is declared infeasible and is penalized in such a way that all unfeasible individuals were worse than any feasible one. Moreover, this penalty is proportional to the number of remaining stations (relative to the total number of stations) after the failure, in order to provide a nice slope of the fitness landscape toward feasibility. For feasible individuals, an average of the total makespan and the sum of the makespans of all partial problems is used: when only the total makespan is used, some individuals start bloating, without much consequence on the total makespan thanks to the final compression that is performed by CPT, but nevertheless slowing down the whole run because of all the useless repeated calls to CPT.

\section{Results on Zeno 10}

For zeno10, all runs found the optimal solution in the very early generations, for both evolution engines $(10+10)-E S$ and $(10,70)-E S$ (rather often, in fact, the initialization procedure produced a feasible individual that CPT could compress to the optimal makespan). 
As already mentioned (see Section 3.2), the number of backtracks used by CPT was in any case limited to a large number to avoid endless searches. However, in order to precisely investigate the simplification due to Divide-andEvolve, we took a closer look at the number of backtracks used by CPT alone, and noticed two things: First, when forbidden to use any backtrack, CPT nevertheless found a suboptimal solution with makespan 915); Second, when searching for the optimal solution without limit on the number of backtracks, CPT never used more than 33 backtracks on a single iteration, with a total of 154 altogether. It was hence decided to try different limits on the number of backtrack per iteration during Divide-and-Evolve procedure, from 35 (above the actual number that is necessary for CPT alone) to 1 (0 was not actually possible there). The results are presented in Table 2 and demonstrate that, in all cases, Divide-and-Evolve was able to drive CPT toward the optimal solution, even though no backtracks could actually be used by CPT. Note, however, that for the most difficult case (limit set to 1 backtrack), the $(10+$ $10)-E S$ engine did not perform very well, while the $(10,70)-E S$ case was much more robust.

Table 2. Performance of Divide-and-Evolve on zeno10 using the $(10+10)-E S$ evolution engine (except last line) when the number of backtracks allowed for CPT is limited.

\begin{tabular}{|c|c|c|c|c|}
\hline Limit & Makespan & Maximal \# BKT & \# Stations & \# Success \\
\hline \hline 35 & 453 & 33 & 5 & $5 / 11$ \\
\hline 20 & 453 & 2 & 20 & $5 / 11$ \\
\hline 10 & 453 & 1 & 5 & $5 / 11$ \\
\hline 1 & 453 & 1 & 6 & $1 / 11(10,10)-\mathrm{ES}$ \\
\cline { 3 - 5 } & & & 6 & $9 / 11(10,70)-\mathrm{ES}$ \\
\hline
\end{tabular}

Here again, when forbidden to use any backtrack, CPT nevertheless found a suboptimal solution with makespan 815); Second, when searching for the optimal solution without limit on the number of backtracks, CPT never used more than 8,066 backtracks on a single iteration, with a total of 27,560 altogether. As with zeno10, different limits on the number of backtrack were run, from 8070 (slightly above the actual number that is necessary for CPT alone) to 1 . The results are presented in Table 2 and again demonstrate that Divide-and-Evolve was indeed able to drive CPT toward the optimal solution, though not when allowed no backtrack at all. Also, for the most difficult case (limit set to 10 backtracks), the $(10+10)-E S$ engine could not find the optimal solution, while the $(10,70)-E S$ case could, with a much smaller number of stations. Note that the $(10,70)-E S$ engine was able to find an optimal solution with no backtrack only once in 11 runs, with more than 50 stations . . 


\section{Results on Zeno 12}

For zeno12 (see Table 3), most runs (around $80 \%$ on average) found the optimal solution. The running time for one generation of the $(10,70)-E S$ engine (70 evaluations) was about $30 \mathrm{mn}$ on a $3.4 \mathrm{GHz}$ Pentium IV processor (because different individuals can have very different number of stations, all running times are rough averages over all runs performed for those experiments). All solutions were found before 20 generations.

Table 3. Performance of Divide-and-Evolve on zeno12 using the $(10+10)-E S$ evolution engine (except last line) when the number of backtracks allowed for CPT is limited.

\begin{tabular}{|c|c|c|c|c|}
\hline Limit & Makespan & Maximal \# BKT & \# Stations & \# Success \\
\hline \hline 8,070 & 549 & 5,049 & 9 & $8 / 11$ \\
\hline 8,000 & 549 & 4,831 & 13 & $4 / 11$ \\
\hline 100 & 549 & 5 & 21 & $2 / 11$ \\
\hline 50 & 549 & 28 & 3 & $2 / 11$ \\
\hline 20 & 549 & 3 & 20 & $3 / 11$ \\
\hline 10 & 549 & $2-1$ & $7-11$ & $6 / 11(10,70)-\mathrm{ES}$ \\
\hline \hline
\end{tabular}

\section{Results on Zeno 14}

A more interesting case is that of zeno14: remember that the present Divideand-Evolve EA uses CPT-2, that is unable to find any solution to zeno14: the results given in table 1 have been obtained using CPT-3. But whereas it proved unable to solve the full problem, CPT-2 could nevertheless be used to solve the hopefully small instances of zeno14 domain that were generated by the Divide-and-Evolve approach - though taking a huge amount of CPU time for that (on average, $90 \mathrm{mn}$ for one generation of 70 evaluations). Note that here, setting a limit on the number of backtracks allowed for CPT was in any case mandatory, to prevent CPT from exploring the too complex cases that would have resulted in a never-returning call (as does a call to the full problem).

The optimal solution (makespan 476) was found in 3 out of 11 runs, with a limit on the number of backtracks set to 120,000 . Note that Divide-andEvolve was unable to find the optimal solution when using a smaller number of backtracks, though it repeatedly found feasible solutions (see Table 4) even with the lowest limit of 1 backtrack (while CPT is not able to find any feasible solution alone, whatever the number of backtracks it is allowed). 
Table 4. Performance of Divide-and-Evolve on zeno14 with limited number of backtracks using the $(10,70)-E S$ evolution engine.

\begin{tabular}{|c|c|}
\hline \hline Limited no BKT & Best Makespan \\
\hline \hline 120,000 & 656 \\
\hline 10,000 & 892 \\
\hline 1,000 & 603 \\
\hline 1 & 868 \\
\hline \hline
\end{tabular}

\section{Discussion on single-objective results}

The main conclusion of those experiments is the proof-of-concept of the Divide-and-Evolve approach. Not only Divide-and-Evolve has been able to find an optimal solution to zeno14 using a version of CPT that was unable to do so, but it also has demonstrated that it could find optimal solutions to a given problem using a very limited setting for CPT. And though, due to the huge overload of CPT calls through Unix forks, it was not possible to see statistically significant decrease in the CPU time needed for different settings of the number of backtracks allowed to CPT, there is no doubt that limiting CPT will allow Divide-and-Evolve to progress more quickly. Further experiments (using CPT3) are however needed to more precisely quantify the gain, and determine the best tradeoff.

\subsection{A multi-objective problem}

\section{Problem description}

In order to test the feasibility of the multi-objective approach based on the Divide-and-Evolve paradigm, we extended the zeno benchmark with an additional criterion, that can be interpreted either as a cost, or as a risk: in the former case, this additional objective is an additive measure, whereas in the latter case (risk) the aggregation function is the max operator.

The problem instance is shown in Figure 1: the only available routes between cities are displayed as edges, only one transportation method is available (plane), and the duration of the transport is shown on the corresponding edge. Risks (or costs) are attached to the cities (i.e., concern any transportation that either lands or takes off from that city). In the initial state, the 3 persons and the 2 planes are in City 0, and the goal is to transport them into City 4.

As can be easily computed (though there is a little trick here), there are 3 remarkable Pareto-optimal solutions, corresponding to traversing only one of the 3 middle cities. Going through City 1 is fast, but risky (costly), whereas going through City 3 is slow and safe and cheap.

When all persons go through respectively City 1, City 2 and City 3, the corresponding values of the makespans and costs in the additive case are ( 8 , 


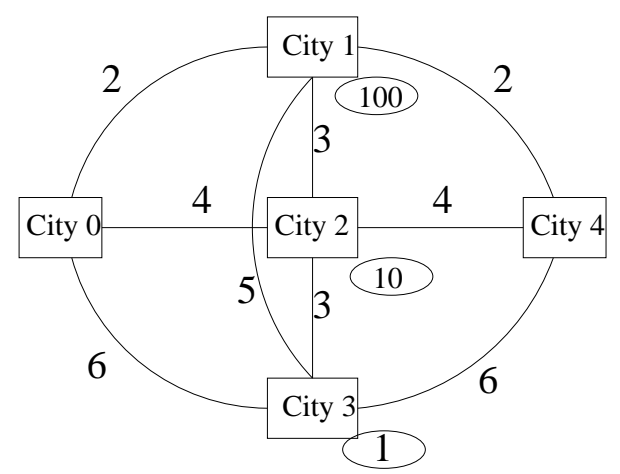

a) The instance: Durations are attached to edges, costs/risks are attached to cities (in gray circles).

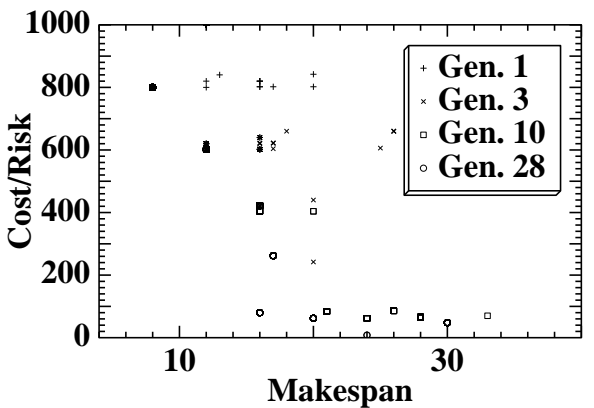

b) The population at different generations for a successful run on the cost (additive) instance of the zeno miniproblem of Figure 1-a.

Fig. 1. The multi-objective Zeno benchmark.

$800),(16,80)$ and $(24,8)$, whereas they are, in the max case, $(8,100)$, $(16,10)$ and $(24,1)$.

\section{Problem complexity}

It is easy to compute the number of possible virtual stations: each one of the 3 persons can be in one of the 5 cities, or not mentioned (absent predicate). Hence there are $3^{6}=729$ possible combinations, and $729^{n}$ possible lists of length $n$. So even when $n$ is limited to 6 , the size of the search space is approx. $10^{17} \ldots$

\section{The algorithm}

The EA is based on the standard NSGA-II multi-objective EA [4]: standard tournament selection of size 2 and deterministic replacement among parents + offspring, both based on the Pareto ranking and crowding distance selection; a population size of 100 evolves during 30 generations. All other parameters were those used for the single objective case.

\section{Fitnesses}

The problem has two objectives: one is the the total makespan (as in the single-objective case), the other is either the risk (aggregated using the max operator) or the cost (an additive objective). Because the global risk only takes 3 values, there is no way to have any useful gradient information when used as fitness in the max case. However, even in the additive case, the same 
arguments than for the makespan apply (section 4.1), and hence, in all cases, the second objective is the sum of the overall risk/cost and the average (not the sum) of the values for all partial problems - excluding from this average those partial problems that have a null makespan (when the goal is already included in the initial state).

\section{Results}

For the additive (cost) case, the most difficult Pareto optimum (going through city 3 only) was found 4 times out of 11 runs. However, the 2 other remarkable Pareto optima, as well as several other points in the Pareto front were also repeatedly found by all runs. Figure 1-b shows different snapshots of the population at different stages of the evolution for a typical successful run: at first ('+'), all individuals have a high cost (above 800); At generation 3 $(' \times$ '), there exist individuals in the population that have cost less than 600; At generation 10 (squares), many points have a cost less than 100. But the optimal $(24,8)$ solution is only found at generation 28 (circles).

The problem in the risk context (the max case) proved to be, as expected, slightly more difficult. All three Pareto optima (there exist no other point of the true Pareto front in the max case) were found only in 2 runs out of 11. However, all runs found both the two other Pareto optima, as well as the slightly sub-optimal solutions that goes only through city 3 but did not find the little trick mentioned earlier, resulting in a $(36,1)$ solution.

In both cases, those results clearly validate the Divide-and-Evolve approach for multi-objective TPPs - remember that CPT has no knowledge of the risk/cost in its optimization procedure - it only aggregates the values a posteriori, after having computed its optimal plan based on the makespan only - hence the difficulty to find the 3rd Pareto optimum going only through city3.

\section{Discussion and Further Work}

First, note that any planner can be used to solve the local problems. In particular, both exact and suboptimal planners are suitable. Some experiments will be made using other planners than CPT. However, because the final goal is to find an optimal plan which join the station of departure and the terminus, using an optimal planner might be mandatory, and, at least, most probably makes things easier for the Evolutionary Algorithm. And because CPT is developed and maintained by one of the authors, we will more likely stick to it in the future.

A primary theoretical concern is the existence of a decomposition for any plan with optimal makespan. At the moment, because of the restriction of the representation to the predicates that are in the goal, some states become impossible to describe. If one of these states is mandatory for all optimal 
plans, the evolutionary algorithm may be unable to find the optimal solution. In the Zeno domain for instance, it can be needed to link a specific person to a specific plane. This may happen when two persons can be indistinctly boarded into two planes, which thus play a symmetrical role between two given stations, but do not play a symmetrical role w.r.t. the overall goal of the problem. The in predicate should then be taken into account when splitting the optimal solution. The main difficulty, however, is to add the corresponding state invariant between at and in (a person is either at a location or in a plane).

The results presented in section 4.1, though demonstrating that indeed Divide-and-Evolve can solve problems that cannot be directly solved by CPT, also show that the search capabilities of the proposed algorithm should be improved for more robustness.

But there is a lot of space for improvements, e.g. on the variation operators: at the moment, both are completely blind, without any use of any domain knowledge. Of course, this is compliant with a "pure" evolutionary approach ...that is also known to be completely inefficient in the case of combinatorial optimization problems. Crossover can be improved by choosing the crossing station in the second parent such that is is close from that of the first parent, at least, at the moment, according to the syntactic distance. The Add mutation, that randomly adds a station in the list, will be improved by building the new station in such a way that it is somehow half-way from both surrounding stations. And the choice of the station to be deleted in the Del mutation will be biased toward the stations that are very easy to reach (in terms of actual number of backtracks used).

Also, all parameters of the algorithm will be carefully fine-tuned.

Of course the Divide-and-Evolve scheme has to be experimented on more examples. The International Planning Competition provides many instances in several domains that are good candidates. Preliminary results on the driver problem showed very similar results that those reported here on the zeno domain. But other domains, such as the depot domain, or many real-world domains, involve (at least) 2 predicates in their goal descriptions (e.g., in and on for depot). It is hence necessary to increase the range of allowed expressions in the description of individuals.

Other improvements will result from the move to CPT-3, the new version of CPT, entirely rewritten in C. It will be possible to call CPT from within the EA, and hence to perform all grounding, pre-processing and CSP representation only once: at the moment, CPT is launched anew for each partial computation, and a quick look at table 1 shows that on zeno14 problem, for instance, the run-time per individual will decrease from 193 to 8 seconds. Though this will not per se improve the quality of the results, it will allow us to tackle more complex problems than even zeno14. Along the same lines, other planners, in particular sub-optimal planners, will also be tried in lieu 
of CPT, as maybe the Divide-and-Evolve approach could find optimal results using sub-optimal planners (as done in some sense in the multi-objective case, see section 4.2).

But deeper improvements will be possible after that move, with respect to problem representation. Because Divide-and-Evolve will have access to all exclusions among predicates that are derived and maintained by CPT, exclusions among predicates might be automatically derived, including exclusions across predicates, such as those involving predicates in and on in the depot domain. Second, and maybe more important, the expressive power of the representation of the stations will be increased: at the moment, only predicates that are listed in the overall goal are considered in the intermediate stations. And the example of zeno14 clearly shows that, though the $D A E$ approach can indeed break the complexity barrier, solving instances that CPT could not directly solve, it will not be able to reach the global optimum with such restriction (one can construct examples where in predicates are necessary to actually optimally break the problem). It is hence planned to allow other predicates to be used to represent intermediate stations. Of course, this will also increase the size of the search space, and some detailed analysis will be needed to somehow determine the minimal set of predicates that are needed for a given problem in order that the $D A E$ approach can find the global optimum.

A last but important remark about the results is that, at least in the single objective case, the best solution found by the algorithm was always found in the early generations of the runs (35 at most for Zeno14): it could be the case that the simple splits of the problem into smaller sub-problems that are done during the initialization are the main reasons for the results. Detailed investigations will show whether or not an Evolutionary Algorithm is actually useful in that context!

Nevertheless, we do believe that using Evolutionary Computation is mandatory in order to solve multi-objective optimization problems, as witnessed by the results of section 4.2, that are, to the best of our knowledge, the first ever results of Pareto optimization for TPPs, and are enough to justify the Divide-and-Evolve approach.

Acknowledgement. Vincent Vidal is supported by the ANR "Planevo" project n JC05_41940.

\section{References}

1. F. Bacchus. The 2000 AI Planning Systems Competition. Artificial Intelligence Magazine, 22(3):47-56, 2001.

2. A. H. Brie and P. Morignot. Genetic Planning Using Variable Length Chromosomes. In 15th Intl Conf. on Automated Planning and Scheduling, 2005. 
3. T. Bylander. The Computational Complexity of Propositional STRIPS planning. Artificial Intelligence, 69(1-2):165-204, 1994.

4. K. Deb, S. Agrawal, A. Pratab, and T. Meyarivan. A Fast Elitist NonDominated Sorting Genetic Algorithm for Multi-Objective Optimization. In M. Schoenauer et al., editor, PPSN'2000, pages 849-858. Springer-Verlag, LNCS 1917, 2000.

5. C. Desquilbet. Détermination de trajets optimaux par algorithmes génétiques. Rapport de stage d'option B2 de l'Ecole Polytechnique. Palaiseau, France, Juin 1992. Advisor: Marc Schoenauer. In French.

6. A.E. Eiben and M. Schoenauer. Evolutionary computing. Information Processing Letter, 82(1):1-6, 2002.

7. A.E. Eiben and J.E. Smith. Introduction to Evolutionary Computing. Springer Verlag, 2003.

8. R. Fikes and N. Nilsson. STRIPS: A New Approach to the Application of Theorem Proving to Problem Solving. Artificial Intelligence, 1:27-120, 1971.

9. M. Fox and D. Long. The Automatic Inference of State Invariants in TIM. Journal of Artificial Intelligence Research, 9:367-421, 1998.

10. M. Fox and D. Long. PDDL2.1: An Extension to PDDL for Expressing Temporal Planning Domains. Journal of Artificial Intelligence Research, 20:61-124, 2003.

11. H. Geffner. Perspectives on Artificial Intelligence Planning. In Proc. AAAI2002, pages 1013-1023, 2002.

12. J. J. Grefenstette. Incorporating Problem Specific Knowledge in Genetic Algorithms. In Davis L., editor, Genetic Algorithms and Simulated Annealing, pages 42-60. Morgan Kaufmann, 1987.

13. W.E. Hart, N. Krasnogor, and J.E.Smith, editors. Evolutionary Computation Special Issue on Memetic Algorithms, volume 12:3. MIT Press, 2004.

14. W.E. Hart, N. Krasnogor, and J.E. Smith, editors. Recent Advances in Memetic Algorithms. Studies in Fuzziness and Soft Computing, Vol. 166. Springer Verlag, 2005.

15. J. Hoffmann, J. Porteous, and L. Sebastia. Ordered Landmarks in Planning. Journal of Artificial Intelligence Research, 22:215-278, 2004.

16. Jörg Hoffmann and Stefan Edelkamp. The Deterministic Part of IPC-4: An Overview. Journal of Artificial Intelligence Research, 24:519-579, 2005.

17. J. Koehler and J. Hoffmann. On Reasonable and Forced Goal Orderings and their Use in an Agenda-Driven Planning Algorithm. JAIR, 12:338-386, 2000.

18. R. Korf. Planning as Search: A Quantitative Approach. Artificial Intelligence, 33:65-88, 1987.

19. D. Long and M. Fox. The 3rd International Planning Competition: Results and Analysis. Journal of Artificial Intelligence Research, 20:1-59, 2003.

20. D. McDermott. PDDL - The Planning Domain Definition language. At http://ftp.cs.yale.edu/pub/mcdermott, 1998.

21. D. McDermott. The 1998 AI Planning Systems Competition. Artificial Intelligence Magazine, 21(2):35-56, 2000.

22. P. Merz and B. Freisleben. Fitness Landscapes and Memetic Algorithm Design. In David Corne, Marco Dorigo, and Fred Glover, editors, New Ideas in Optimization, pages 245-260. McGraw-Hill, London, 1999.

23. N. J. Radcliffe and P. D. Surry. Fitness variance of formae and performance prediction. In L. D. Whitley and M. D. Vose, editors, Foundations of Genetic Algorithms 3, pages 51-72. Morgan Kaufmann, 1995. 
24. J.L. Schlabach, C.C. Hayes, and D.E. Goldberg. FOX-GA: A Genetic Algorithm for Generating and Analyzing Battlefield Courses of Action. Evolutionary Computation, 7(1):45-68, 1999.

25. Marc Schoenauer, Pierre Savéant, and Vincent Vidal. Divide-and-evolve: a new memetic scheme for domain-independent temporal planning. In J. Gottlieb and G. Raidl, editors, Proc. EvoCOP'06. Springer Verlag, 2006.

26. D. Smith and D. S. Weld. Temporal Planning with Mutual Exclusion Reasoning. In Proceedings of IJCAI-99, pages 326-337, 1999.

27. L. Spector. Genetic Programming and AI Planning Systems. In Proc. AAAI 94, pages 1329-1334. AAAI/MIT Press, 1994.

28. V. Vidal and H. Geffner. Branching and Pruning: An Optimal Temporal POCL Planner based on Constraint Programming. In Proceedings of AAAI-2004, pages 570-577, 2004.

29. V. Vidal and H. Geffner. Branching and Pruning: An Optimal Temporal POCL Planner based on Constraint Programming (to appear). Artificial Intelligence, 2005.

30. D. S. Weld. An Introduction to Least Commitment Planning. AI Magazine, 15(4):27-61, 1994.

31. D. Whitley, T. Starkweather, and D. Fuquay. Scheduling problems and traveling salesman: The genetic edge recombination operator. In J. D. Schaffer, editor, Proc. $3^{\text {rd }}$ Intl Conf. on Genetic Algorithms. Morgan Kaufmann, 1989. 